Cahiers de la recherche sur les droits

Cahiers

Fur les Droits fondamentaux

9 | 2011

Conseil constitutionnel et droits fondamentaux

\title{
Du mythe de « l'augustinisme politique » de Carl Schmitt
}

\section{Wagdi Sabète}

\section{(2) OpenEdition}

1 Journals

Édition électronique

URL : https://journals.openedition.org/crdf/5455

DOI : $10.4000 /$ crdf.5455

ISSN : 2264-1246

Éditeur

Presses universitaires de Caen

Édition imprimée

Date de publication : 1 décembre 2011

Pagination : 127-142

ISBN : 978-2-84133-396-7

ISSN : $1634-8842$

Référence électronique

Wagdi Sabète, «Du mythe de "l'augustinisme politique » de Carl Schmitt », Cahiers de la recherche sur les droits fondamentaux [En ligne], 9 | 2011, mis en ligne le 01 décembre 2012, consulté le 15 novembre 2022. URL : http://journals.openedition.org/crdf/5455 ; DOI : https://doi.org/10.4000/crdf.5455 


\title{
Du mythe de "l'augustinisme politique" de Carl Schmitt
}

\author{
Wagdi SABÈTE
}

Maître de conférences en droit public (HDR) à la faculté de droit et de science politique de I'Université de La Rochelle

I. La théologie politique et l'absence d'augustinisme politique chez Schmitt

A. La médiation institutionnelle de l'État avatar de la médiation chrétienne?

B. L'État: médiateur de réalisation d'un droit idéal?

C. La notion d'ennemi politique, transposition du concept théologique d'hérétique?

II. Les conséquences de la sécularisation sur la théorie juridique de Schmitt: le détournement schmittien de saint Augustin
A. La valeur de la justice comme philosophie politique augustinienne
B. Le concept d'augustinisme juridique ou politique proprement dit
C. Précautions de la recherche généalogique

\begin{abstract}
Si la République est la Chose du Peuple, et s'il n'y a point peuple quand il n'y a pas association sous un droit consenti or, il n'y a pas droit où il n'y a pas justice, il suit indubitablement qu'où il n'y a pas justice, il n'y a pas République ${ }^{1}$.

Tous les concepts prégnants de la théorie moderne de l'État sont des concepts théologiques sécularisés. Et c'est vrai non seulement de leur développement historique, parce qu'ils ont été transférés de la théologie à la théorie de l'État - du fait, par exemple, que le Dieu tout-puissant est devenu législateur omnipotent -, mais aussi de leur structure systématique, dont la connaissance est nécessaire pour une analyse sociologique de ces concepts ${ }^{2}$.
\end{abstract}

Lieu commun, malentendu, erreur répandue, ou évidente vérité? La pensée politique de Carl Schmitt est influencée par le christianisme, et son œuvre manifeste les «stigmates $»^{3}$ du catholicisme. Saint Augustin fut « son maître à penser ${ }^{4}$ et Théorie de la décision est d'une inspiration augustinienne $e^{5}$. Aussi curieux que cela puisse paraître, dans sa préface de l'ouvrage de Schmitt, le professeur Olivier Beaud écrit: «Bien que dans la présentation de sa généalogie intellectuelle, Schmitt se réfère notamment à Savigny et à Hegel, son véritable maître à penser est plutôt saint Augustin, ou plus exactement, la tradition augustiniste dont il est très consciemment l'héritier. ${ }^{6}$

1. Saint Augustin, La Cité de Dieu, t. III, liv. XIX, chap. XXI.

2. C. Schmitt, Théologie politique [2 ${ }^{\mathrm{e}}$ édition de 1934], Paris, Gallimard (NRF), 1992, p. 46.

3. Le mot, me semble-t-il, est approprié au contexte.

4. O. Beaud, préface de l'ouvrage de C. Schmitt, Théorie de la Constitution, Paris, PUF (Quadrige), 2008, p. 49 [désormais O. Beaud, préface]. Cet ouvrage est aussi édité dans la collection Léviathan en 1993.

5. O. Beaud, préface, p. 25 et 49. On ne saurait oublier les travaux de S. Baume sur C. Schmitt. Voir, par exemple, Carl Schmitt, penseur de l'État. Genèse d'une doctrine, Paris, Presses de la Fondation nationale des sciences politiques, 2008.

6. O. Beaud, préface, p. 49 . 
De façon plus nuancée mais tout aussi affirmative, on a pu lire dans la littérature qui trace la généalogie de l'œuvre schmittienne qu' "après 1947, Carl Schmitt retourne au christianisme, comme bon nombre de conservateurs, et présente son autodéfense ${ }^{7}$, ou encore que «l'ensemble de son œuvre, sa théorie du droit comme sa théorie politique, se tient sous les auspices de l'Église romaine ${ }^{8}$ et ainsi, il «a maintenu son rapport au catholicisme et a défendu la place de l'Église dans l'État national-socialiste» ${ }^{9}$.

C'est ainsi qu'on a présenté l'origine de la pensée de Schmitt ${ }^{10}$ au point que certains constitutionnalistes ont créé un nouveau concept qui frappe les spécialistes de saint Augustin ${ }^{11}$ : «l'augustinisme juridique» ${ }^{12}$. Pourtant, pour chacun de ces derniers, et sans exception, il y a bien des années-lumière qui séparent l'œuvre de saint Augustin de la doctrine de Schmitt. Il y en a bien d'autres qui ne permettent ni de qualifier de «juridique» ${ }^{13}$ - au sens entendu par les juristes modernes - une seule idée chez saint Augustin, ni de prétendre qu'il existe un augustinisme politique ${ }^{14}$ au sens envisagé par cette thèse. On pourrait à la rigueur soutenir l'existence d'un augustinisme politique ${ }^{15}$ pendant certaines périodes de l'histoire médiévale (IX ${ }^{\mathrm{e}}$ siècle en particulier), mais il serait inconcevable d'envisager une quelconque influence de cet augustinisme politique au cours des $\mathrm{XIX}^{\mathrm{e}}$ et $\mathrm{XX}^{\mathrm{e}}$ siècles, si l'on comprenait bien la pensée et l'œuvre de saint Augustin. À supposer que le terme recouvre une réalité politique déterminée, cette réalité a cessé d'exister après le IX ${ }^{\mathrm{e}}$ siècle en Europe. D'où l'étonnement d'user de ce terme pour qualifier la doctrine de Schmitt. Notre interrogation ici relève autant de la recherche en théorie politique et constitutionnelle qu'en histoire des idées politiques. Il est nécessaire de formuler d'emblée trois précisions: d'abord, loin de nous l'idée d'une étude apologétique qu'un regard superficiel se contentant des dehors de l'article peut supposer trop hâtivement. Loin de nous également l'intention d'ajouter quelques éléments sur une notion bien capitale dans la pensée chrétienne, telle que la justice, comme une lecture rapide peut le suggérer. Et, enfin, il n'est pas dans notre intention de mettre en perspective l'état des lieux de la pensée politique de certains auteurs chrétiens sur les questions de l'État, son rôle, le gouvernement ou la démocratie. Il ne faut donc pas se tromper de projet: le nôtre consiste humblement à vérifier l'exactitude de l'existence d'un lien philosophique ou conceptuel entre la doctrine de Schmitt et celle de saint Augustin. Un tel projet est académique à bien des égards: d'abord, parce qu'il vise à éclairer un débat très important sur le lien entre la pensée théologique et certaines théories politiques, ensuite il montre en filigrane les effets néfastes de la sécularisation sur la pensée politique et constitutionnelle moderne, et, enfin, il touche à un problème non résolu en droit public: à savoir la dogmatique en droit public. Notre seul objectif, mais aussi notre modeste ambition ici, est donc de vérifier l'exactitude de l'assertion suivante: les idées de Schmitt trouvent leur origine dans la pensée de saint Augustin et spécialement dans son œuvre principale La Cité de Dieu.

Pourquoi une telle interrogation? Parce que si l'on considère la position des spécialistes de la pensée augustinienne ${ }^{16}$ sur cette question, et bien qu'ils ne soient guère spécialistes de Schmitt, leur compréhension de l'œuvre de saint Augustin en particulier, et de la doctrine chrétienne en général, nous incite à être nuancé avant d'affirmer la moindre filiation généalogique entre les doctrines de Schmitt et de saint Augustin. Et si la démonstration qui suit vise à déterminer la nature réelle d'un lien supposé, sans être véritablement démontré par la doctrine qui l'affirme, c'est précisément dans le but de s'interroger sur des concepts tels que l'augustinisme politique ou juridique dont la contradiction réside dans le terme même. On se borne à étudier deux questions fondamentales qui marquent la théorie de Schmitt et au regard desquelles le problème de l'inspiration augustinienne a été posé par des spécialistes de la théorie constitutionnelle: d'une part, l'absence d'augustinisme politique dans la pensée de Schmitt et donc l'écart entre sa théologie politique et la doctrine de saint Augustin. Et d'autre part, le détournement de l'œuvre de saint Augustin par Schmitt et cela nous montre les conséquences de la sécularisation des concepts théologiques. Les deux volets fondamentaux de la théorie de Schmitt, que sont la théorie de la décision et la théorie de l'État médiateur, ont été perçus comme ayant un « ancrage théologique». À ce double titre, il serait important de clarifier, dans un premier temps, la nature réelle des liens qui rattachent ces concepts à l'œuvre de saint Augustin sur les théories de l'institution, de la décision et de l'ennemi politique (I), ensuite il faudrait essayer, dans un second temps, d'analyser l'origine de ce travail de sécularisation

7. D. Cumin, Carl Schmitt: biographie politique et intellectuelle, Paris, Les éditions du CERF, 2005, p. 187.

8. Ibid., p. 176 .

9. Ibid., p. 177. Dans son ouvrage, David Cumin a suivi - avec plus de nuance qu'Olivier Beaud - la même thèse en admettant que Carl Schmitt «se tourne - en leur empruntant non sans les déformer - vers ou contre des auteurs aussi différents que saint Augustin, saint Thomas d'Aquin et les Pères de l'Église [...] » (p. 22) ; mais il affirme aussi que la théorie de l’État total est marquée par « un système de pensée théologique et catholique» (p. 176-177). Voir aussi sa thèse de doctorat, Carl Schmitt, 1888-1985, Université Lyon III, 1996.

10. O. Beaud, préface.

11. H. de Lubac, "Augustinisme politique? ", Théologies d'occasion, Paris, Desclée de Brouwer, 1984, p. 255-308.

12. O. Beaud, préface, p. 49.

13. Sur le sens et le contenu de l'augustinisme, voir le remarquable ouvrage du professeur H.-I. Marrou, Saint Augustin et l'Augustinisme, Paris, Seuil (Points sagesses), 2003

14. H.-X. Arquillière, L'augustinisme politique: essai sur la formation des théories politiques du Moyen Âge [1934], Paris, Vrin, 2006.

15. Ibid., p. 146

16. P. Sellier, Pascal et saint Augustin, Paris, Albin Michel, 1995; Y. Floucat, Maritain ou le catholicisme intégral et l’humanisme démocratique, Paris, Pierre Téqui éditeur (Questions disputées), 2003, notamment p. 83-111; L. Jerphagnon, Augustin et la sagesse, Paris, Desclée de Brouwer, 2006, notamment chap. VIII, p. 75-81; A. Marc, Raison philosophique et Raison révélée, Paris, Desclée de Brouwer, 1955, notamment p. 128-138. 
chez Schmitt qui consiste en le détournement de saint Augustin par Schmitt (II).

\section{La théologie politique et l'absence d'augustinisme politique chez Schmitt}

L'affirmation selon laquelle saint Augustin est le «maître à penser» de Schmitt nécessite de notre part une vérification sur trois questions fondamentales qui caractérisent la théorie de Schmitt: la médiation institutionnelle de l'État est-elle vraiment à l'image de la médiation de l'Église (A) ? Cette médiation viserait-elle à réaliser un droit idéal comme celle de l'Église qui viserait la réalisation de la Cité de Dieu (B) ? Mais si l'État ou l'unité politique doit se constituer, c'est précisément avec l'ami et contre l'ennemi tout comme l'Église se constitue autour de la communauté des croyants (C). Car la notion d'ennemi politique trouverait selon $\mathrm{Schmitt}$ son équivalent théologique dans la notion d'hérétique.

\section{A. La médiation institutionnelle de l'État avatar de la médiation chrétienne?}

L'interprétation faite par une partie de la doctrine constitutionnaliste française de l'œuvre de Schmitt selon laquelle "l'investiture divine du Christ en tant que sujet médiateur est née d'un acte de volonté, d'une décision, et non pas d'une application automatique de la loi divine ${ }^{17}$ et donc «La décision et la médiation sont inextricablement liées » ${ }^{18}$, nous invite à la plus grande prudence pour deux raisons: la première, parce que «le penseur de la Médiation ${ }^{19}$ ne l'a pas "pensé » comme un théologienphilosophe tel que saint Augustin a su le faire. La rupture entre le concept de la médiation schmittienne et celui de la médiation chrétienne doit être démontrée, même si cela nous oblige à exposer ici quelques éléments d'ordre théologique qu'on n'a pas souhaité exposer ${ }^{20}$. Théologiquement, le mystère de l'Incarnation, considérée par cette thèse comme source d'inspiration de Schmitt, ne procède pas d'une décision volontaire qui interviendrait à un moment donné selon le choix d'une volonté et pour des raisons qui la justifieraient, comme cette thèse l'affirme. La théologie chrétienne ne considère pas que le Christ est le produit d'une volonté qui l'a fait exister (sinon ce serait la même volonté qui a fait exister Dieu Lui-même, alors laquelle ${ }^{21}$ ?). L'intervention du Christ dans l'histoire des hommes, selon la foi chrétienne, n'est pas une réponse à une norme ou une loi divine, moins encore une exécution, une expression ou une traduction d'une décision unilatérale, comme l'avaient cru Schmitt et ceux qui soutiennent l'inspiration chrétienne de sa doctrine. La décision, si décision était, n'est pas prise à un moment donné dans le temps, mais peut être avant le temps ${ }^{22}$. L'investiture divine du Christ en tant que sujet médiateur n'est pas née d'un acte de volonté, comme cela a été écrit ${ }^{23}$, à l'image de la volonté humaine, mais peut être d'un état de fait et d'une nature personnelle dont la connaissance relève du mystère théologique qui ne concerne, en aucun cas, la rationalité de la pensée politique et constitutionnelle. L'union de la nature divine et de la nature humaine dans la personne du Christ est au cœur de la foi chrétienne et elle a un nom: le mystère de la Trinité ${ }^{24}$. Ce n'est donc pas un acte de volonté qui serait à l'origine d'une décision située et déterminée dans le temps et par le temps comme un regard simpliste l'affirme ${ }^{25}$. Contrairement à la médiation schmittienne, celle du christianisme ne constitue pas un moment, ni une introduction à l'œuvre du salut, moins encore une décision. Elle est elle-même le salut offert à l'homme non seulement selon saint Augustin, mais selon la foi chrétienne en général. L'analogie de Schmitt ne se base que sur la forme que peut revêtir l'institution, sans tenir compte de la finalité de celle-ci, d'où le caractère formaliste de cette thèse.

Ensuite, toujours selon Schmitt, la médiation originale est répétée par l'Église. Celle-ci est la manifestation de Dieu dans l'Incarnation. Schmitt envisage l'État de la même manière: il transpose les concepts théologiques uniques et mystiques sur sa théorie de l'État où celui-ci sera bâti sur le modèle de cette Église. Ces concepts transposés n'appartiennent pas spécialement à saint Augustin. Or, il donne à l'État, selon sa compréhension du rôle de l'Église, le rôle de médiation entre le droit et l'individu. Tout comme le rôle de médiation entre Dieu et les hommes passe par l'Église, celui de l'État se situe entre le droit et

17. C’est ainsi que le professeur Olivier Beaud a exprimé la pensée de Schmitt: préface, p. 57.

18. Ibid.

19. L'expression est celle du professeur Olivier Beaud: préface, p. 56.

20. Nous devons avouer le réel dilemme de l'auteur de ces lignes: d'une part, il fallait répondre au besoin du lecteur qui n'a pas de familiarité particulière avec la doctrine chrétienne, mais d'autre part, il fallait éviter l'écueil de faire une étude apologétique ou théologique qui s'éloigne de notre objectif (vérifier l'exactitude de la thèse doctrinale qui fait de Schmitt un augustinien). Il nous importe peu finalement que cette thèse soit soutenue par Schmitt lui-même ou par une partie de la doctrine constitutionnaliste publiée en France.

21. Naturellement, il n'existe aucune réponse, pas même théologique, à cette question.

22. D'ailleurs, elle dépendait du consentement d'une femme: Marie (non pas de la volonté arbitraire et absolue de Dieu). Marie (sujet concerné par la décision) a rendu possible cette incarnation. C'est peut-être ici d'ailleurs que réside une autre différence entre le fonctionnement du pouvoir décisionnel des hommes et celui de Dieu d'un point de vue théologique et chrétien. Face au choix de la liberté humaine, Dieu de saint Augustin n'est pas tout-puissant comme l'État total schmittien, mais plutôt impuissant et désarmé. C'est le contraire de ce que Schmitt conçoit par la puissance décisionnelle et ses effets sur les individus.

23. Selon la représentation de Schmitt par O. Beaud, préface, p. 57.

24. Voir l'Évangile de Jean, I, versets 1 et 14: «Au commencement était le Verbe, et le Verbe était Dieu [...] Et le Verbe s'est fait chair, et il a habité parmi nous".

25. O. Beaud, préface. 
l'individu et passe par les institutions. Tout comme Dieu s'est incarné dans le Christ, l'Église aussi doit se doter d'un corps, c'est-à-dire d'une institution ${ }^{26}$. Il en est de même pour l'État qui se dote désormais d'une institution afin d'assurer cette médiation. Tout comme l'Église regroupe une communauté de fidèles, l'État regroupe une «communauté politique». L'analogie est surtout erronée: car si l'Église est une instance de médiation entre deux ordres divin et humain, on voit mal pourquoi une autre instance (l'État) jouerait le même rôle sans évacuer totalement celui de l'Église ? Cela irait à l'encontre de la thèse augustinienne. Étant donné que la médiation de l'Église est établie historiquement et théologiquement, il reste à justifier la médiation de l'État. Or, celle-ci se justifie par le rôle de l'État dans la réalisation d'un droit idéal appliqué aux individus. D'où la différence avec saint Augustin : la pierre fondatrice de la cité de Dieu est l'amour (et certainement pas la souveraineté ni le monopole de produire le droit positif). Cette pierre est portée par l'Église, médiatrice de la cité de Dieu, c'est pourquoi la relation entre les deux cités traduit une relation entre le temporel et le spirituel représenté par l'Église. Ces deux cités s'opposent car l'une est créée par l'amour alors que l'autre est créée par la haine ou l'amour-propre. L'une est tournée contre l'ennemi (Schmitt) et l'autre vers Dieu. Elles vivent côte à côte, mais, en même temps, elles se compénètrent. La séparation des deux cités sera définitive le jour du Jugement dernier (selon saint Augustin, livre XXII). Les peuples, les États, et les empires passent les uns après les autres mais la cité de Dieu subsiste ${ }^{27}$. En quoi donc cette vision purement métaphysique serait la source inspiratrice de la théorie de l'État de Schmitt? Et si l'Église joue le rôle de médiateur entre les deux ordres, selon le raisonnement de Schmitt, quel est donc le rôle de l'État? Celuici doit avoir un autre rôle distinct de celui de médiateur entre l'ordre divin et l'ordre humain. À vrai dire, il n'y a aucune raison - ni logique ni théologique - de répéter la médiation à l'infini: d'abord, il y a celle du Christ, ensuite celle de l'Église, et enfin celle de l'État. Pourquoi, donc, ne pas étendre ce devoir théologique de médiation répétée à d'autres unités infra- ou supra-étatiques (les grands espaces, les empires) ? Pourquoi la communauté internationale ou les régions, ainsi que d'autres unités politiques, ne peuvent être, elles aussi, l'expression d'une médiation divine? Sinon, pourquoi ne pas répéter la médiation chrétienne, comme si celle du Christ n'était pas suffisante? Et dans ce cas, pourquoi ne pas reconnaître que, pour «le chrétien Schmitt» ${ }^{28}$, la médiation du Christ ne suffit pas et même l'Église est aussitôt remplacée par un État total? Si l'on considérait, avec O. Beaud, que «la théorie schmittienne de l'État recevait son éclairage ultime dans ce devoir théologique de répéter la médiation ${ }^{29}$, on irait à l'encontre de toutes les sources chrétiennes connues qui n'envisagent ni devoir, ni même nécessité, à répéter une telle médiation. Non seulement, selon la doctrine catholique, l'État n'est pas un médiateur valable devant Dieu, mais l'idée même d'un devoir de répéter la médiation est une idée antichrétienne par définition, dans la mesure où elle suggère l'insuffisance de la médiation du Christ. Nous ne sommes plus dans le cadre des idées chrétiennes bien connues. Quant à l'Église chez Schmitt, elle est aussitôt remplacée par l'État qui assure désormais, en tant qu'institution, la «traduction matérielle» et le "passage du transcendant invisible à l'empirique, de l'irrationnel au rationnel $»^{30}$.

\section{B. L'État: médiateur de réalisation d'un droit idéal?}

Ceci est le point de départ de la théorie du droit de Schmitt. Il propose une idée dépourvue de toute originalité, à savoir l'existence d'un droit idéal et d'un droit réel, ou plutôt réalisé: ce droit idéal, le vrai droit, dépend dans sa réalisation de l'État dont émane le droit positif (expression du droit idéal). Considérer que «la doctrine juridique de Schmitt épouse largement les contours de la doctrine catholique de l'État et du Droit», et que «son originalité réside dans le diagnostic, assez augustinisant, de la transformation du droit supra-positif, lorsque celui-ci tend à devenir du droit positif-étatique ${ }^{31}$, est purement et simplement une erreur qui révèle une profonde méconnaissance ou au moins une incompréhension de l'œuvre de saint Augustin. Et en voici la démonstration. D’abord, la thèse de l'augustinisme politique de Schmitt est fondée sur une conception erronée des deux cités. Car, rien n'est aussi étranger aux deux cités de saint Augustin que le dualisme de Schmitt (droit idéal / droit réel). D’une part, ce dualisme n'a rien de spécialement catholique, et d'autre part, il est sans rapport particulier avec La Cité de Dieu. L'idée d'une opposition entre le droit de la cité et le droit naturel existe depuis Sophocle. La même idée a fait son chemin à l'époque chrétienne et même sous la Révolution française en ayant plusieurs acceptions étymologiques et philosophiques. On ne saurait ignorer la portée polysémique de l'expression «droit naturel». Aux

26. S. Baume, Carl Schmitt, penseur de l'État..., p. 205.

27. G. Bardy, Saint Augustin, l'homme et l'œuvre, 6e édition, Paris, Desclée de Brouwer, 1946, p. 514-515.

28. L'expression est utilisée par le professeur Stéphane Rials en désignant Carl Schmitt. Voir son article "Le chrétien Schmitt et le juif Jésus », Droits Revue française de théorie juridique, $\mathrm{n}^{\circ}$ 40, 2004, p. 163-172. Mais la qualification de "chrétien", précédant ainsi le nom d'un auteur, a déjà été utilisée, et à juste titre, bien avant l'article de S. Rials pour désigner un autre auteur de la même époque (Georges Bernanos né en 1888 comme Schmitt) dans la première édition française de 1956 de l'ouvrage de H. U. von Balthasar, Le chrétien Bernanos [réédition: M. de Gandillanc (trad.), Les Plans (Suisse), Parole et Silence, 2004]. Voir également sur l'engagement politique de Schmitt: O. Beaud, «Contre Schmitt? Carl Schmitt, juriste nazi ou juriste qui fut nazi. Tentative de réexamen critique», Droits. Revue française de théorie juridique, ${ }^{\circ}$ 40, $2004, \mathrm{p} .207-218$.

29. O. Beaud, préface, p. 58 .

30. S. Baume, Carl Schmitt, penseur de l'État..., p. 213-215.

31. O. Beaud, préface, p. 33 . 
$\mathrm{XIX}^{\mathrm{e}}$ et $\mathrm{XX}^{\mathrm{e}}$ siècles, les juristes jus-naturalistes ont voulu limiter l'action de l'État par les principes du droit naturel afin que le droit positif soit encadré par une certaine idée de la justice. Il s'agit ici d'une variante classique de la théorie du droit naturel qui n'a aucune filiation augustinienne spécifique. Par ailleurs, Schmitt oppose une norme juridique et une norme de réalisation du droit. Celle-ci traverse tout le droit: il s'agit d'une règle objective d'action (ou de réaction) ${ }^{32}$. La dictature souveraine voit l'état de choses auquel elle entend mettre fin par son action. Elle ne suspend pas la constitution en vertu d'une norme conforme à la constitution, mais elle cherche à instaurer l'état de choses pour invoquer une constitution à établir. Elle échappe ainsi à toute considération juridique. Il s'agit donc d'une pure et simple question de puissance ${ }^{33}$ et d'état de fait. Il poursuit: "Toute instance temporelle s'efface comme c'est le cas de la dépendance du souverain par rapport à Dieu. Cromwell a invoqué Dieu pour "sa mission" [...] il n'a pas laissé planer le moindre doute sur le fait qu'il voyait en Dieu la source de son pouvoir.» ${ }^{34}$

Le concept de la dictature souveraine invoque le pouvoir constituant qui ne peut être aboli par aucune constitution s'opposant à lui. Ce pouvoir n'est pas un organe, ni même le peuple en tant que corps constitué et absorbé dans l'État, mais une idée qui domine la théorie de l'État de Schmitt. Comment ne pas y voir les ombres de l'idéalisme allemand? Dieu, pour Schmitt, est un commettant distinct de ce titulaire du pouvoir constituant ${ }^{35}$. Inutile de dire que ce n'est pas ainsi que saint Augustin voit le rôle de Dieu dans l'histoire des hommes. Saint Augustin n'a pas soutenu la thèse selon laquelle dans la cité des hommes (Rome), on trouve la félicité ou la justice parfaite, ni par l'application de certaines valeurs, ni par une dictature qui lutte contre le désordre, ni par une décision politique souveraine mettant fin au chaos. Bien au contraire, il a réfuté ces doctrines en montrant, d'une part, les vains efforts pour réaliser, en cette vie, une félicité parfaite, et, d'autre part, il nous a montré en quoi consiste la félicité du chrétien, ce qu'elle peut être dans la vie présente et ce qu'on peut espérer dans la vie future ${ }^{36}$. L'espérance de saint Augustin, contrairement à celle de Schmitt, n'est pas en Rome. Elle est ailleurs, dans une autre cité: Jérusalem ${ }^{37}$ ! Son espérance donne l'idée d'un ailleurs et avec elle le courage de s'y rendre. L'espérance chez Schmitt est ici et maintenant sans ailleurs ni avenir. Pour un constitutionnaliste comme Schmitt, l'espérance réside dans les institutions et les décisions politiques prises. Pour saint Augustin, et pour d'autres auteurs chrétiens, l'Espérance réside dans la grâce. La simple lecture d'un Pascal, d'un Maritain, ou d'un Bernanos, nous offre un éclairage de l'idée de la grâce ${ }^{38}$. Celle-ci ne risque pas d'être retenue par l'idéalisme des Lumières germaniques.

Enfin, contrairement à ce qui est écrit au sujet du «fossé entre le droit positif et le droit naturel [qui] conduit à l'acceptation résignée des lois injustes et traduit l'opposition $^{39}$ des deux Cités terrestre et divine et l'impossible conciliation entre justice divine et justice humaine», saint Augustin offre une vision plus nuancée que la vision schématisante et simpliste du rapport entre les deux cités. Il offre aussi une conception du rapport entre justice divine et justice humaine qui ferait de la première un principe directeur de la seconde ${ }^{40}$. La vision augustinienne de ces deux cités fera l'objet d'analyse dans les lignes suivantes. Mais il faut souligner d'abord, qu'en intitulant son œuvre La Cité de Dieu, saint Augustin use d'une expression bien connue des Romains qui évoque une fondation. Cette fondation n'oppose pas forcément toujours et systématiquement Rome et l'Église, mais les deux cités sont «idéales quasi platoniciennes ${ }^{41}$ dont les réalisations concrètes ne peuvent être que contingentes, ou deux principes vitaux qui dominent la cité des hommes. C'est précisément ici que la conception de Schmitt ne doit rien à celle de saint Augustin: le principe selon lequel l'homme vit pour posséder et dominer, et le principe spirituel «l'homme vit selon Dieu ${ }^{42}$ n'ont pas grand-chose à voir l'un avec l'autre. Ces deux principes de vie, que nous appelons mystiquement deux cités, ne peuvent être confondus dans leur essence. En un mot, saint Augustin traite de la condition humaine d'un point de vue spirituel, et Schmitt traite aussi de la condition humaine mais d'un point de vue purement politique. Saint Augustin donne à l'histoire, contrairement à Schmitt, avec cette proposition de deux cités, un principe directeur, un principe éclairant le jugement et non pas un principe-solution à court terme, au mal d'un Empire païen ou d'une république finissante ${ }^{43}$. L'État ou la cité terrestre pour saint Augustin n'est pas un instrument de réalisation d'un droit idéal comme l'avaient envisagé les juristes du droit naturel, mais les hommes de la

32. C. Schmitt, La Dictature, Paris, Seuil, 20oo, p. 141

33. Ibid., p. 143 .

34. Ibid.

35. Ibid., p. 144

36. Voir notamment le livre XIX sur le Souverain Bien de La Cité de Dieu.

37. Sur l'étude du rapport entre les deux cités, voir le professeur J.-M. Trigeaud, «Rome et Jérusalem: le mythe des deux cités», Persona ou la Justice au double visage, Gênes, Studio Editoriale di Cultura (Nuova Biblioteca Filosofia Oggi), 199o, p. 226-239.

38. Sur cette notion chez Pascal et saint Augustin, voir P. Sellier, Pascal et saint Augustin, p. 222-357.

39. O. Beaud, préface, p. 34 .

40. Ibid.

41. Voir l'introduction de J.-C. Eslin à La Cité de Dieu, liv. I à X, Paris, Seuil, 1994, p. 8.

42. Saint Augustin, De Civitate Dei, liv. XV, chap. 1.

43. Nous pensons en particulier à la république de Weimar, voir O. Beaud, Les derniers jours de Weimar. Carl Schmitt face à l'avènement du nazisme, Paris, Descartes \& $\mathrm{C}^{\mathrm{ie}}$, 1997. 
cité doivent vivre selon la vérité à laquelle ils adhèrent. Il ne s'agit pas d'appliquer une loi divine idéale à la cité des hommes, mais de transformer la vie intérieure de ces hommes pour qu'elle soit la meilleure possible. Ceci est une démonstration de la divergence philosophique fondamentale de l'esprit de ces deux auteurs et de leurs deux mondes. Chez saint Augustin, la valeur n'est pas donnée à l'État (Rome), tandis que chez Schmitt, son hostilité pour l'individualisme le pousse quasiment à minimiser et à réduire la valeur de l'individu et à glorifier celle de l'État ${ }^{44}$. Or, «réduire la valeur de l'individu à l'accomplissement de son devoir», comme l'exprime Sandrine Baume $^{45}$, comporte, à notre avis, une trace de la philosophie kantienne et certainement pas augustinienne. Son concept d' «État total», quelle que soit l'acception ${ }^{46}$ qu'il lui donne (qualitative ou quantitative), ne doit rien non plus à La Cité de Dieu, comme nous allons le voir à la fin de cette démonstration. La dimension spirituelle de la cité de Dieu est totalement absente chez Schmitt: cette cité se dévoile, comme l'a très bien écrit le professeur JeanMarc Trigeaud, en tant que "principe secret de la cité des hommes; même si celle-ci la bafoue ou la méprise, comme elle est le ressort caché de notre nature. Elle en marque les exigences: elle est le siège ultime des valeurs et de la valeur suprême du juste ${ }^{47}[. .$.$] Elle le présente dans sa personne$ et reflète ainsi la personne divine qui vit en chacun ${ }^{48}$.

Bien que certaines lectures de Schmitt affirment le caractère métaphysique de sa doctrine, nous pensons le contraire: Schmitt s'oppose à des concepts métaphysiques tels que la justice, l'équité, la paix, alors que saint Augustin les considère comme condition sine qua non de la politique, de la république et du droit. La métaphysique de Schmitt, comme sa théologie, servent la politique, non pas les choses de l'âme ou de la spiritualité. Rien n'est plus antimétaphysique qu'une telle attitude intellectuelle. À cet égard, il est intéressant de noter la proximité entre Carl Schmitt et Hans Kelsen. Cela d'ailleurs a déjà été observé ${ }^{49}$, à juste titre, parce que ces concepts n'ont aucune réalité propre pour les deux auteurs (quoi qu'on en dise du catholicisme de Schmitt). Pour Schmitt, ces valeurs ne signifient rien en dehors du contexte politique où elles seront utilisées. Pour Kelsen, il s'agit d'une position plutôt épistémologique qui se justifie par un relativisme axiologique radical favorisé par l'adhésion à la philosophie de David Hume ${ }^{50}$.

À bien des égards, la conception augustinienne des deux cités reste inconciliable avec celle de Schmitt. En premier lieu, le livre XIX est l'un des plus célèbres livres de La Cité de Dieu. Il s'agit de lier étroitement dans ce livre le sort de l'une et l'autre cité, la terrestre et la céleste; non pas d'un lien extérieur et simplement chronologique, comme au livre XVIII, mais en tissant subtilement, par le biais d'une réflexion sur la cité et la Res publica, ce qu'il veut dire de la valeur et de la limite de la politique. C'est un livre éminemment politique, considéré comme référence classique des philosophes politiques, et qui représente, pour les spécialistes, l'orientation chrétienne de la politique. Au chapitre XXI, on trouve un passage décisif qui montre à quel point sa doctrine diverge de celle de Schmitt (non positiviste selon sa propre qualification), mais aussi de celle des auteurs positivistes. Pour saint Augustin, il n'y eut jamais une République romaine, au sens entendu chez certains philosophes, car l'ordre politique de Rome ne fut jamais la chose du peuple (définition exacte de la république). Le peuple est une association qui repose sur la sanction d'un droit consenti et sur la communauté d'intérêts. Or, un droit consenti dans une république implique que celle-ci ne peut être gouvernée sans la justice. Certes, il reconnaît que l'État de Rome est une république, et que le peuple romain est un peuple, mais il ajoute dans le chapitre XXIV que dès les premiers temps et aux époques suivantes, il y eut des passions et corruptions auxquelles ce peuple s'est livré, et des sanglantes séditions pour mener des guerres sociales et civiles qui ont brisé la concorde et la paix. Et saint Augustin résume sa conception en une seule phrase: «il n'y a ni peuple ni république où il n'y a pas justice». La justice, pour lui, est la vertu qui permet de rendre à chacun ce qui lui appartient. En second lieu, ce qui sépare à l'origine les deux cités est ceci: «Deux amours ont bâti deux cités: celle de la terre par l'amour de soi jusqu'au mépris de Dieu, celle du ciel par l'amour de Dieu jusqu'au mépris de soi. L'une se glorifie en elle-même, l'autre dans le Seigneur. L'une en effet demande sa gloire aux hommes; l'autre tire sa plus grande gloire de Dieu [...] L'une, dans ses puissants, chérit sa propre force; l'autre dit à son Dieu: je t'aimerai, Seigneur, toi ma force. ${ }^{51}$

En troisième lieu, le thème de la Cité de Dieu constitue dans l'œuvre de saint Augustin une métaphore littéraire qui, si elle renvoie à l'idée de citoyenneté dans la religion civile des Romains, échappe cependant à toute analogie avec l'État et la cité des hommes. La cité de Dieu se rapporte à la sagesse, à la Paix, au culte unique de Dieu, à l'Église, à la providence divine, à la justice là où elle est pratiquée, mais en même temps, la cité de Dieu n'est jamais dite entièrement dans une seule des choses qu'elle

44. Voir l'analyse de S. Baume de l'ouvrage La valeur de l'État et la signification de l'individu, écrit en 1914, dans Carl Schmitt, penseur de l'État..., p. 21 et 162.

45. Ibid., p. 162

46. Sur ces acceptions, voir S. Baume, Carl Schmitt, penseur de l'État..., p. 69.

47. J.-M. Trigeaud, «Rome et Jérusalem: le mythe des deux cités», p. 234

48. Ibid., p. 239

49. R. Kolb, préface, Deux textes de Carl Schmitt. La question clé de la Société des Nations. Le passage au concept de guerre discriminatoire, Paris, Pedone, 2009, p. 9.

50. Le professeur R. Kolb a noté à juste titre que les deux auteurs « ont commencé leur carrière par une critique de la science juridique comme dernier dépositaire, séculaire de concepts et de véhicules de pensée théologiques» (ibid.).

51. Saint Augustin, La Cité de Dieu, t. XIV, liv. XXVIII, chap. I. 
peut désigner. Elle est présente sur la terre mais elle y est en exil. Les deux cités sont donc à la fois distinctes et mélangées, et ce jusqu'à la fin des temps ${ }^{52}$. Mais dans le même temps, la cité terrestre aspire à la paix et elle en est capable. La cité céleste rapporte la paix céleste sans la confondre avec ce qui peut être désigné par erreur la paix véritable par la cité terrestre ${ }^{53}$. La cité céleste est autonome par rapport à la cité terrestre mais elle n'y est pas indifférente. Les deux cités luttent entre elles, mais elles sont appelées à vivre dans la concorde. La référence à la cité de Dieu n'est légitime que si cette cité est susceptible d'être décelée au fondement même de la cité terrestre qui peut la trahir ou la rejeter. Le rapport entre les deux cités vise, ainsi, à distinguer et à articuler ce que l'on a désigné, selon les époques, comme le temporel et le spirituel, le Trône et l'Autel, l'Église et l'État, ou ce que nous appellerions aujourd'hui le religieux et le politique. Certaines lectures politiques de saint Augustin devraient tenir compte de la distinction qu'il convenait de faire entre pouvoir politique et religion au début du IV ${ }^{\mathrm{e}}$ siècle, sinon elles laisseraient penser que saint Augustin a voulu unir ce qui était séparé alors qu'il semble plutôt s'agir de l'inverse. Les analogies successives de Schmitt vont hélas dans ce sens qui n'a jamais été voulu par saint Augustin. En quatrième lieu, pour saint Augustin, le mélange et la distinction des deux cités dureront jusqu'à la fin des temps. En conséquence, il ne sera possible de mettre en place un pouvoir civil qui puisse se confondre avec la cité de Dieu. Ceci est pourtant le point qui fonde la théologie de Schmitt et qui le sépare radicalement de la doctrine de saint Augustin. Un spécialiste de l'œuvre de saint Augustin, tel Lucien Jerphagnon, ne saurait confondre le rapport entre les deux cités comme le fait un constitutionnaliste dépourvu des outils nécessaires à la compréhension de l'œuvre et de l'expérience intérieure de saint Augustin: «La cité de Dieu ne confère à ses ressortissants ni extraterritorialité, ni immunité diplomatique, pas même, pour garder l'analogie, la double nationalité», écrit le professeur L. Jerphagnon ${ }^{54}$. Plus qu'une théorie politique destinée à donner forme aux institutions politiques de l'État, $L a$ Cité de Dieu, mais aussi Les Confessions, parlent d'un combat qui a lieu au plus intime de la conscience de chacun pour la primauté de l'amour de Dieu confronté à certaines formes de l'amour de soi ${ }^{55}$. C'est peut-être ici, d'abord, que réside la différence entre un philosophe chrétien comme saint Augustin et un idéologue intellectuel animé par une soif de puissance comme Schmitt. C'est encore ici que réside la différence qui sépare les moyens de transformation politique et constitutionnelle et les moyens de transformation de la Conscience personnelle dans le for intérieur de chacun. C'est, enfin, ainsi qu'on voit la différence qui marque la cité des hommes, Rome, de Jérusalem. La première n'a pas connu la paix mais la guerre, la seconde ne connaît que la paix de l'âme et la concorde. Cette question assure la transition pour le problème de l'ennemi politique.

\section{La notion d'ennemi politique, transposition du concept théologique d'hérétique?}

Si les deux cités sont mêlées l'une dans l'autre, c'est justement parce que les adversaires de la cité de Dieu aujourd'hui peuvent être ses citoyens demain, et inversement: certains, qui prennent part aujourd'hui à ses sacrements, ne seront peut-être pas associés à sa gloire future. En un mot: pour saint Augustin, l'ennemi de ce jour peut être l'ami de demain, alors que pour Schmitt, une chose est sûre: l'État se constitue, à l'origine, autour de l'ami et contre l'ennemi qu'il faut anéantir. Celui-ci n'aurait aucune chance d'être ami un jour. Ceci est une autre manifestation de la divergence majeure avec les thèses chrétiennes, et surtout augustiniennes. Il convient de se référer à l'analyse critique conduite par Jacques Maritain à l'encontre du dualisme catégorique ami-ennemi chez Schmitt.

C'est bien l'analyse qui s'inspire de ce que saint Augustin a écrit dans La Cité de Dieu non pas celle de Schmitt. Bien que certains spécialistes de la théorie constitutionnelle pensent qu'il existe une "opposition structurale au sein de la pensée catholique entre l'hétérodoxie augustiniste et l'orthodoxie aristotélico-thomiste ${ }^{56}$, notre objectif est de démontrer le contraire, au moins, sur certains sujets. Ainsi la référence à Maritain n'est pas neutre. Elle se justifie par la volonté de répondre à ce lieu commun selon lequel la rupture entre Schmitt et Maritain se fondait sur le fait que le premier est soi-disant augustinien, alors que l'autre est aristotélicien et thomiste ${ }^{57}$. La divergence entre ces deux auteurs consiste, entre autres ${ }^{58}$, dans le concept adopté de la souveraineté: pour Maritain, "les deux concepts de Souveraineté et d'Absolutisme ont été forgés sur la même enclume. Ils doivent être ensemble mis au rebut $»{ }^{59}$. Ce propos se situe aux antipodes des idées maîtresses de Schmitt à propos de la souveraineté de l'État. Celle-ci constitue le point de divergence majeur d'avec les idées politiques de Maritain. La cause de l'opposition entre les deux hommes évoquée par O. Beaud nous

52. Ibid., t. XVIII, liv. LII, chap. IV.

53. Ibid., liv. XIX, chap. XVII

54. L. Jerphagnon, «Introduction», Saint Augustin, La Cité de Dieu, in Euvres, t. II, Paris, Gallimard (Pléiade), 200o, p. XX.

55. Ibid.

56. Voir O. Beaud, préface, p. 49.

57. Ibid.

58. Il y a une opposition majeure entre les deux hommes au sujet de la position de chacun face aux juifs. Sur cette question, il convient de citer P. Vidal-Naquet, Jacques Maritain, l'impossible antisémitisme, précédé de Jacques Maritain et les juifs, Paris, Desclée de Brouwer, 2003.

59. Pour se rendre compte de l'ampleur de cette divergence sur le concept de la souveraineté, voir J. Maritain, L'Homme et l'État, préface de P. Valadier, Paris, Desclée de Brouwer, 2009, p. 47-71. 
semblerait aussi inexacte pour d'autres raisons: d'une part, sans doute Maritain le philosophe est thomiste et ses écrits philosophiques et théologiques le démontrent, mais il est aussi, et au même titre, augustinien en tant que chrétien engagé et penseur politique. Après quinze années de thomisme épistémologique, Maritain fut conduit par les événements politiques en France à s'engager dans le débat théologico-politique lors de la condamnation par le cardinal Andrieu, archevêque de Bordeaux, le 25 août 1926, du quotidien maurassien. C'est entre 1926 et 1928, que ses écrits politiques apparaissent profondément marqués par des sources augustiniennes. Ses publications sont d'un très grand intérêt pour la réflexion politique des catholiques ${ }^{60}$. On y voit clairement les influences de saint Augustin. Il suffit donc de connaître l'ensemble de son œuvre et notamment ses écrits politiques pendant cette époque pour se rendre compte à quel point il fut politiquement augustinien et non seulement philosophiquement thomiste ${ }^{61}$. D'autre part, Schmitt tourna le dos entièrement aux grands principes chrétiens et surtout à l'augustinisme, contrairement à ce qu'on pouvait écrire sur ce point. Les idées développées ici montrent bien cette divergence. Pour ne prendre que l'exemple de l'hérétique politique traité dans l'ouvrage de Maritain, L'Homme et l'État ${ }^{62}$, force est d'y voir la parfaite démonstration d'une telle divergence entre les thèses augustiniennes de Maritain et celles de Schmitt sur la définition même de l'hérétique politique et l'attitude adoptée à son égard ${ }^{63}$. Saint Augustin considère la peine civile, dans les cas de l'hérétique religieux, comme remède menant celui-ci à se repentir ${ }^{64}$. Quant à Maritain, il écrit que: «Dans la société du Moyen Âge, l’hérétique était celui qui brisait l'unité religieuse. Dans une société laïque d'hommes libres, l'hérétique est celui qui brise les croyances et les pratiques démocratiques communes, celui qui prend position contre la liberté, ou contre l'égalité fondamentale des hommes, ou encore contre la dignité et les droits de la personne humaine. ${ }^{65}$

À la vérité, l'opposition entre Maritain et Schmitt découle de la divergence intellectuelle et doctrinale entre les deux hommes: le premier est profondément chrétien engagé qui a parfaitement compris le sens du message spirituel du christianisme en se soumettant avec humilité à ce qui le dépasse tandis que le second est un intellectuel extrémiste qui a laissé l'idéalisme subvertir le message chrétien sans faire preuve d'humilité spirituelle ${ }^{66}$. Les autres divergences en découlent. Maritain élabora une pensée politique subtile, parfois fort éloignée du néothomisme ${ }^{67}$, où se mêlent subordination stricte du pouvoir temporel au spirituel et apologie de la sécularisation et de la liberté religieuse. Et c'est notamment sur la liberté religieuse que Maritain s'éloigne de saint Augustin au soir de sa vie ${ }^{68}$. Mais Maritain s'éloigne de Schmitt aussi et de façon plus radicale encore sur la question de la souveraineté. Il est hostile à toutes les formes temporelles de la souveraineté : étatique, populaire ou nationale ${ }^{69}$. C'est précisément la méconnaissance de son œuvre sur cette question qui explique la raison de l'effacement d'une autre opposition à Schmitt. Celle-ci n'est pas une simple traduction de l'opposition entre le thomisme (représenté par Maritain) et l'augustinisme (représenté par Schmitt). Car l'augustinisme de Maritain découle principalement, et entre autres, de sa position sur la question de la souveraineté. C'est cela qui fait de lui un héritier de l'augustinisme politique médiéval. Les spécialistes de Maritain estiment que le thomisme et l'augustinisme sont loin d'être deux doctrines irréductiblement antagonistes et ainsi elles peuvent fort bien se marier dans la pensée d'un intellectuel catholique comme Maritain ${ }^{70}$. Il est d'ailleurs fort intéressant de connaître le rôle que l'œuvre de saint Augustin a pu jouer dans la formation de saint Thomas d'Aquin lors de son séjour à Paris pour nuancer la radicale opposition entre les deux Pères de l'Église ${ }^{71}$. La démarche rationnelle et philosophique de saint Thomas d'Aquin doit, en effet, à saint Augustin ce que ce dernier appelle intellectus fidei ${ }^{72}$.

Un dernier point à rectifier : selon l'interprétation du professeur O. Beaud, «si Schmitt use de la méthode historique en analysant les notions constitutionnelles, c'est moins pour découvrir l'esprit du peuple que pour examiner les effets de la lutte politique sur le destin des institutions juridiques ${ }^{73}$.

60. G. de Thieulloy, Antihumanisme intégral? L'augustinisme de Jacques Maritain, Paris, Pierre Téqui éditeur, 2006, p. 54-61.

61. Sur l'augustinisme de Jacques Maritain, voir G. de Thieulloy, Antihumanisme intégral?... ; J. Maritain, Le Crépuscule de la civilisation, Montréal, Éditions de l'Arbre, 1941; J. Maritain, L'Homme et l'État.

62. Ibid., p. 137-142.

63. Ibid., p. 138

64. P. Christophe, «Hérésie», Petit dictionnaire de l'histoire de l'Église, Paris, Desclée de Brouwer, 1994, p. 63.

65. J. Maritain, L'Homme et l'État, p. 138.

66. En ce qui concerne Maritain, il s'agit bien d'un homme qui passa les dernières années de sa vie chez les Petits Frères de Jésus où il mourut en 1973. À partir de 1971, Jacques Maritain vécut chez les Petits Frères de Jésus à Toulouse.

67. Sur les questions de l'État, du droit et de la politique selon saint Thomas d'Aquin, voir M. Villey, Questions de saint Thomas sur le droit et la politique, Paris, PUF, 1987; «La théologie de Thomas d'Aquin et la formation de l'État moderne», in Théologie et Droit dans la science politique de l'État moderne (Actes de la table ronde organisée par l'École française de Rome avec le concours du CNRS, à Rome, 12-14 novembre 1987), Rome, École française de Rome, 1991, p. 31-49.

68. G. de Thieulloy, Antihumanisme intégral?..., p. 152-154.

69. Ibid., p. 103

70. Ibid., p. 154

71. Et par là même le thomisme de Maritain et le prétendu augustinisme de Schmitt.

72. Sur l'apport de saint Augustin à la formation théologique de saint Thomas d'Aquin et sur la notion de l'intelligence de la foi, voir M.-S. Gillet, Thomas d'Aquin, Paris, Dunod (Les constructeurs), 1949, p. 22-53.

73. O. Beaud, préface, p. 92 . 
Si cette affirmation est parfaitement exacte, il n'est pas pour autant vrai qu'on puisse l'établir sur ce qu'on appelle «la philosophie augustinienne de l'histoire» qui interdirait à Schmitt l'évolutionnisme d'un Savigny. Faite de ruptures, l'histoire selon Schmitt obéit à la dialectique de la lutte entre l'ami et l'ennemi. C'est précisément ici qu'on ne voit aucun lien avec une quelconque "philosophie augustinienne de l'histoire ${ }^{74}$. Car rien dans les écrits de saint Augustin ne permet d'affirmer une telle assertion. Et voici la démonstration:

En premier lieu, le fondement de l'unité politique de la nation chez Schmitt est de se constituer contre l'ennemi mais aussi avec et pour l'ami ${ }^{75}$. C'est précisément ici que réside un autre point de divergence avec le christianisme. La théorie de Schmitt sur ce point nous donne une idée de l'attitude de l'Empire paien, et non point celle qui est recommandée par le christianisme. Toutes les descriptions de Schmitt nous aident à découvrir l'essence de la politique païenne et les fondements de l'Empire païen qui se constitue contre un autre, contre son ennemi. Il est vrai, écrit Maritain, que pour cette politique-là, séparée de la morale, le sommet de l'intelligence politique serait le discernement de l'ennemi. «Et voilà pourquoi les totalitarismes vont d'eux-mêmes à la guerre intérieure ou extérieure, en vertu d'une loi métaphysique beaucoup plus puissante que la volonté des hommes d'État. C'est la loi mystique de l'esprit du monde, que la haine subjugue l'amour et qu'on n'aime le sien que dans la mesure où on hait les autres. ${ }^{76}$

Et comment ne pas voir la contradiction entre un concept tel que la guerre menée contre l'ennemi dont la désignation appartient à un seul homme qui incarne souverainement la décision de la nation, et un commandement chrétien comme celui-ci: «Aimez vos ennemis, bénissez ceux qui vous maudissent, faites du bien à ceux qui vous haïssent, et priez pour ceux qui vous maltraitent et qui vous persécutent ${ }^{77}$. On voit dans cette citation une opposition radicale entre le sens de l'ennemi selon la source biblique et les vues de l'Empire païen telles qu'un penseur comme Schmitt les avait envisagées. Notons aussi que l'ennemi pour Schmitt ne saurait être qu'un ensemble d'individus groupés, un "ennemi public», hostis, par opposition à l'ennemi privé, inimicus. Cet ennemi public doit être identifié à un État étranger qui menace la souveraineté et l'intégrité territoriale d'un autre État. L'ennemi chez Schmitt est toujours la figure d'une altérité radicale, qu'il faut réduire, ou expulser de l'unité politique. Il suffit, en revanche, de lire Emmanuel Levinas et les sources philosophiques judéo-chrétiennes (Le personnalisme chrétien en particulier) pour se rendre compte à quel point l'épiphanie du visage de l'Autre dans le christianisme est située aux antipodes de l'altérité schmittienne. Si Schmitt utilise délibérément des expressions telles que hostis, inimicus, et cette antique opposition entre hostis et inimicus, c'est parce qu'il croit que ces termes ne sont pas galvaudés par des effets d'idéologie qui confondent délibérément la concurrence des intérêts privés, les oppositions abstraites, doctrinales ou partisanes, et la véritable nature de l'antagonisme politique, afin d'en nier la spécificité. Schmitt vise par là un antagonisme concret, lié à une situation concrète dont la logique ultime est la configuration ami-ennemi, et la réponse est la guerre. En second lieu, même si l'on s'écarte du texte biblique, pour se borner strictement aux écrits de saint Augustin dans La Cité de Dieu, celui-ci adopte une conception de l'ennemi située exactement aux antipodes de celle de Schmitt. Il écrit clairement: "Voilà ce que mon Dieu m'a déclaré au sujet de mes ennemis: ne les tuez pas [...] Dieu a montré à l'Église la grâce de sa miséricorde, parce que selon l'apôtre, leurs faux pas [les ennemis] sont le salut des Gentils.» Mais saint Augustin, le philosophe et le croyant, ajoute qu' "il ne suffirait pas de dire "ne les tuez pas", car s'ils étaient renfermés dans leur pays et non répandus à tous lieux, l'Église est partout, pourrait-elle les avoir chez toutes les nations pour témoins des prophéties qui ont annoncé Jésus-Christ » ${ }^{78}$.

Quelle que soit la nuance subtile qui distingue hostes d'inimicus, saint Augustin n'envisage pas les rapports politiques sous le prisme de la nuance dans la nature de l'hostilité. Arriver à ce stade de la démonstration, la même interrogation subsiste: où est l'inspiration augustinienne dans les idées de Schmitt?

\section{Les conséquences de la sécularisation sur la théorie juridique de Schmitt: le détournement schmittien de saint Augustin}

Si certains auteurs estiment que la théorie du droit de Schmitt trouve son ancrage dans « une théologie dès lors qu'elle a pour fondement de validité l'incarnation divine» ${ }^{79}$, on oublie pour autant que cet auteur n'a tiré aucune conséquence de cette incarnation qui constitue l'acte fondateur du christianisme, surtout dans les deux volets de sa théorie: la décision chez Schmitt ne correspond pas à la « décision» divine volontaire de donner sa vie à d'autres personnes,

74. Ibid.

75. Voir C. Schmitt, La Notion de politique. Théorie du partisan, M.-L. Steinhauser (trad.), préface de J. Freund, Paris, Flammarion (Champs), 1992, p. 63-114. Le même ouvrage est d'abord paru dans la collection «Liberté de l'Esprit», créée par R. Aron: Berlin, Dunker \& Humblot, 1963.

76. J. Maritain, Le Crépuscule de la civilisation, p. 55.

77. Matthieu, V, 43-46.

78. Saint Augustin, La Cité de Dieu, t. III, liv. XVIII, chap. XLVI

79. F. Scholz, «Die Theologie Carl Schmitts», in Der Fürst dieser Welt. Carl Schmitt und die Folgen, J. Taubes (dir.), Munich, Schöningh, 1983, p. 165, cité par O. Beaud, préface, p. 49, note 226, où il ajoute que «cet ancrage théologique vaut aussi bien pour l'institution, les deux pôles de la théorie schmittienne». 
et les institutions chez Schmitt ne réalisent en aucun cas ni la paix ni la félicité des hommes selon saint Augustin. Cela nous invite à présenter ici trois raisons supplémentaires pour lesquelles nous réfutons la thèse de l'inspiration augustinienne de Schmitt et nous affirmons, en revanche, le détournement de saint Augustin par Schmitt.

D'abord, la valeur de justice est le fondement de la philosophie du droit et de la politique chez saint Augustin (A) ; ensuite, le concept de l'augustinisme politique proprement dit existe bel et bien mais il n'existe pas dans les théories de Schmitt (B) ; et, enfin, il est nécessaire de prendre une précaution méthodologique avant toute recherche de généalogie intellectuelle entre deux auteurs (C). C'est peut-être cela la leçon principale qu'on peut tirer de cette étude.

\section{A. La valeur de la justice comme philosophie politique augustinienne}

Voilà d'abord ce qui distingue radicalement saint Augustin de Schmitt et de Kelsen à la fois. D'une part, pour saint Augustin, mais aussi pour la pensée chrétienne tout entière, aucune institution politique terrestre ne donnera aux hommes une paix réelle et véritable, aucun système ou décision politique ne confère la joie parfaite que le Christ enseigne. Si l'on définit la république selon Cicéron, c'està-dire la chose du peuple ${ }^{80}$, «il n'y eut jamais une République romaine, car le cadre politique de Rome ne fut jamais la Chose du peuple» ${ }^{81}$. Saint Augustin se réfère à la définition de Scipion selon laquelle un peuple est « une association nombreuse qui repose sur la sanction d'un droit consenti et sur la communauté d'intérêts». Or, ce que Scipion entend par «droit consenti » dans une république ne peut se concevoir sans la justice. Car aucun gouvernement ne peut être sans la justice. «Donc, où il n'y a pas une véritable justice, le droit ne peut être. Car ce qui se fait avec droit se fait aussi avec justice et ce qui se fait sans la justice ne peut se faire avec le droit. ${ }^{82}$

C'est l'absence de cette valeur que saint Augustin reprocha à Rome. En parlant de cité terrestre, il visa le monde de l'époque (Rome) tel qu'il était, avec ses institutions, son histoire, ses gouvernants, ses bonheurs et ses malheurs. Cette cité terrestre est bâtie sur l'amour de soi comme idolâtrie. Ce n'est donc pas seulement une cité comme une ville, un empire ou un mode de gouvernement, mais c'est aussi une disposition intérieure de l'homme. Saint Augustin n'a jamais approuvé l'application des lois injustes ou iniques pour conserver la paix civile, comme cela a été justifié par Schmitt ${ }^{83}$. Au contraire, saint Augustin attribue la ruine de Rome à sa ruine morale qui a précédé sa ruine matérielle. La corruption et l'injustice furent, selon saint Augustin, l'origine et la cause des malheurs publics: il introduit dans le chapitre XXI de son livre I une interrogation aussi célèbre que déstabilisante, qui sera reprise dans le livre XIX: «Y a-t-il encore un peuple, une cité, une république là où il n'y a plus de vraie justice? » De ce point de vue, les deux doctrines se séparent, non seulement sur ce point mais aussi sur l'importance particulière que saint Augustin accorde à l'idée d'une justice liée à une certaine conception du Bien, ce qui traduit en effet une divergence de fond entre la vérité morale de saint Augustin et la vérité politique de Schmitt. Pour Schmitt, la force de l'État est la solution de tout désordre qui règne dans toutes les affaires. Seul un État extrêmement fort pourrait résoudre le terrible désordre y compris non étatique. L'État total ${ }^{84}$ est justement celui qui peut augmenter sa force d'impact. Cela implique bien sûr que l'État se désengage dans certains domaines pour se consacrer à l'essentiel ${ }^{85}$ (à savoir sa propre force et sa puissance). Nous n'avons point l'intention de développer davantage la conception schmittienne de l'État total.

Les concepts théologiques chrétiens ont été simplifiés et isolés de leur finalité et de leur contexte pour servir en quelque sorte de "boîte à outils intellectuels", comme s'il n'y avait pas dans la pensée laïque moderne de concepts pertinents pour construire une théorie juridique ou politique. Et c'est précisément ici que réside l'erreur première de la sécularisation: dans le passage de l'engagement chrétien ou religieux à l'engagement politique ou intellectuel incompris ${ }^{86}$. C'est souvent d'ailleurs la même erreur qui caractérise le nationalisme catholique: croire que la vérité chrétienne peut s'imposer et triompher spectaculairement sur terre et que le Royaume (Cité) de Dieu ${ }^{87}$ peut s'établir par des moyens constitutionnels et institutionnels; ou par le biais d'un certain choix d'organisation politique, sociale ou législative, ou pire encore par une simple décision politique d'un certain contenu qui mettrait fin au chaos. Contrairement à un Villey conscient de cette évidence qui découle de sa foi chrétienne ${ }^{88}$, Schmitt

80. De Republica, I. 25-29

81. Saint Augustin, La Cité de Dieu, t. III, liv. XIX, chap. XXI.

82. Ibid.

83. Selon certaines interprétations, Schmitt ne fait ici qu'adopter la conception de saint Augustin sur ce point, voir O. Beaud, préface, p. 53. Or les écrits de saint Augustin ne semblent pas accréditer cette thèse: il suffit de lire son livre V de La Cité de Dieu où il relève le défi d'interroger Rome (cité terrestre) en ce qu'elle présente de plus cher et plus noble: la recherche de la gloire et de la vertu. Il dit que la république soutient les vices de ses gouvernants.

84. Sur l'État total dans la pensée de C. Schmitt, voir l'ouvrage de S. Baume, Carl Schmitt, penseur de l'État..., p. 82-83.

85. Ibid.

86. C'est souvent d'ailleurs la même erreur commise par les auteurs nationalistes.

87. On peut aussi dire Cité de Dieu pour utiliser le titre de l'ouvrage considéré comme source d'inspiration de Carl Schmitt.

88. «Jésus n'a pas connu le succès, mais la Croix; toute satisfaction de son œuvre, tout système clos, tout cercle parfait, tout discours qui emporte l'adhésion unanime de l'auditoire, tout cela va contre notre nature et la vérité» (M. Villey, Réflexions sur la philosophie et le droit. Les Carnets, Paris, PUF, 1995, liv. IV-45). 
aurait un peu de peine à voir sa vérité crucifiée et humiliée. Une certaine arrogance l'aurait-elle empêché de franchir le pas vers une humilité augustinienne réelle ${ }^{89}$ ? Ou plutôt un certain idéalisme allemand nourrissait sa culture par des éléments étrangers à la culture chrétienne qui aurait fait le reste? Pour saint Augustin, la vérité qui se dégage du christianisme, contrairement à ce qu'on pouvait écrire ou penser, n'aura ni le privilège du succès, ni le triomphe de la victoire, mais tout au plus l'humiliation de la Croix. C'est son sort et son seul privilège. La «vérité» chrétienne que Schmitt, mais aussi d'autres nationalistes catholiques, me semble-t-il, avaient cru devoir défendre est, selon eux, une vérité haut de gamme, et mérite, à ce titre, et pour cette raison, d'être défendue, vu son caractère précieux, alors que celle du christianisme est hors gamme, elle est seule, pauvre, méprisée, et sans défense, et pour cette raison justement elle mérite d'être défendue. Elle est celle que Michel Villey qualifia de "consciente d'avoir hors de soi et non pas en soi l'amour, la vie et la vertu» ${ }^{90}$. Par conséquent, et malgré la séduisante construction de la théorie de la décision, aucune félicité réelle ne triomphe dans notre monde grâce à une théorie, constitutionnelle ou juridique. Il ne suffit pas, selon le christianisme, d'établir l'ordre dans une cité pour que celle-ci puisse accéder au bonheur de la paix et à la sérénité de l'âme raisonnable dont parla saint Augustin. Refuser cela, c'est rejeter l'idée même de la Croix qui est au cœur de la foi chrétienne. Rêver d'un Jérusalem céleste sur terre grâce à une décision politique et à une institution humaine, ce n'est certainement pas s'inspirer de La Cité de Dieu, mais c'est plutôt aller à l'encontre des idées élémentaires exprimées clairement dans l'œuvre de saint Augustin. Ignorer le sens de ses écrits et, au même titre, les implications de la foi qui les anime, c'est exprimer une naïveté déconcertante. Encore une fois, on voit mal comment saint Augustin pourrait inspirer les idées de Schmitt.

\section{B. Le concept d'augustinisme juridique ou politique proprement dit}

Qu'entendons-nous par cette expression? La thèse de «l'augustinisme politique» n'appartient ni à Schmitt, ni à ses contemporains et commentateurs, ni au professeur
O. Beaud. Elle ne remonte pas aux Moyens Âges, mais à 1934 lors de la parution des travaux érudits du chanoine Henri-Xavier Arquillière sur Grégoire VII - Saint Grégoire VII. Essai sur sa conception du pouvoir pontifical $^{91}$-, ainsi que de son ouvrage intitulé L'augustinisme politique. Essai sur la formation des théories politiques du Moyen Âge $e^{92}$. Et même si Schmitt se considère, à tort, comme l'héritier de saint Augustin, il convient de nuancer son affirmation. Car, malheureusement, les héritiers de saint Augustin n'ont pas hérité de son génie. Comme le constate à juste titre H.-X. Arquillière - véritable auteur de la thèse de l'augustinisme politique et créateur de ce concept -, « ils [les héritiers de saint Augustin] ont souvent appauvri et simplifié ses conceptions, notamment en matière politico-religieuse ${ }^{93}$. Schmitt, à notre sens, est l'un de ces derniers. Si l'on peut comprendre l'affirmation unilatérale de Schmitt lui-même selon laquelle sa théorie politique et constitutionnelle transpose des idées théologiques dont l'origine réside dans la théologie chrétienne ${ }^{94}$, on a, en revanche, un peu de peine à suivre les auteurs constitutionnalistes français qui se livrent, sans connaissance suffisante de l'œuvre de saint Augustin, à une telle affirmation ${ }^{95}$. Les spécialistes de saint Augustin affirment une vision du monde (les deux cités) inconciliable avec celle de Schmitt ${ }^{96}$. Il suffit de citer les travaux d'Henri de Lubac ou d'autres spécialistes ${ }^{97}$ pour relativiser l'analogie ou l'interprétation hâtive sur l'existence d'augustinisme politique dans la doctrine de Schmitt. Si le mythe de l'augustinisme politique a pu se maintenir, c'est au prix d'une contradiction et d'une confusion de langage comme l'avait constaté à juste titre Henri de Lubac: «Il n'aurait pu même naître sans l'idée conçue a priori d'un "augustinisme" et d'un "aristotélisme" constituant deux blocs rivaux dont le heurt ou la succession seraient comme le fond intellectuel de l'histoire politique de l'Église en Occident. Mais il n'aurait pas non plus trouvé dans cette histoire ce qui a paru être sa force explicative s'il n'avait également procédé d'une mauvaise lecture de la grande œuvre de saint Augustin. ${ }^{98}$

C'est précisément en raison de cette «lecture» que cette étude a essayé de clarifier le sens de la doctrine de saint Augustin afin de démontrer à quel point celle de Schmitt s'en éloigne radicalement. Il convient de se référer

89. C’est précisément la «souveraineté de la haine», comme disait, à juste titre, Jacques Maritain, qui traduit la rupture entre ces deux hommes et certainement pas le fait que Maritain soit thomiste alors que Schmitt serait augustinien comme cela a été injustement écrit!

90. Ibid., liv. I, $\$ 18$

91. H.-X. Arquillière, Saint Grégoire VII. Essai sur sa conception du pouvoir pontifical, Paris, Vrin, 1934.

92. H.-X. Arquillière, L'augustinisme politique. Essai sur la formation des théories politiques du Moyen Âge, Paris, Vrin, 1934 [réédition: 1956 et 2006 ]

93. Ibid., p. 52.

94. Voir C. Schmitt, Théologie politique, I, p. 42 et 46.

95. Nous insistons sur la nécessité scientifique et académique de connaître les deux auteurs qui font l'objet de l'étude, non pas un seul. Il ne s'agit pas bien sûr d'exiger une connaissance parfaite de tous les philosophes de tous les temps mais de connaitre bien les écrits de l'auteur qu'on considère comme source d'inspiration d'un autre, surtout si la pensée de ce dernier ne prête $a$ priori à aucune généalogie, ni à quelconque similitude ou filiation avec son prétendu «maître à penser».

96. J. Maritain, Le Crépuscule de la civilisation, p. 55.

97. Voir son ouvrage collectif avec G. Chantraine, P. Descourtieux et M. Figura, Augustinisme et Théologie moderne, in CEuvres complètes, t. VIII, Paris, Cerf, 2009; H.-I. Marrou, Saint Augustin et l'Augustinisme.

98. H. de Lubac, "Augustinisme politique? ", p. 264. 
à l'auteur de ce concept, Henri-Xavier Arquillière ${ }^{99}$ : il s'agit d'une doctrine de l'État face à l'Église. Le droit naturel de l'État d'exister indépendamment de l'Église est l'idée maîtresse de cet augustinisme politique. Il s'agit donc d'un droit naturel de l'État vis-à-vis de l'Église. Or, saint Augustin avait une tendance, au moins aux yeux de ses contradicteurs, à absorber l'ordre naturel dans l'ordre surnaturel et le droit de l'État dans celui de l'Église ${ }^{100}$. Il reconnaît, certes, la valeur légitime de l'État chez les anciens Grecs, les Égyptiens, et dans toutes les nations antiques. Mais il a fait prévaloir une tendance, fort bien analysée par Étienne Gilson, à absorber l'ordre naturel dans l'ordre surnaturel. Cette tendance constitue, écrit l'auteur du concept, l'essence de l'augustinisme politique $^{101}$. Il n'est donc pas question de réclamer le droit naturel de l'État face à d'autres États, comme pourrait le concevoir Schmitt à travers la souveraineté internationale de l'État, moins encore face aux ressortissants nationaux, mais il s'agit pour saint Augustin de reconnaître ce droit d'exister juste vis-à-vis de l'Église. Et même s'il s'agit d'un droit naturel de l'État à l'existence, il faudrait encore que deux conditions soient réunies: la première, que l'État se fonde sur la justice, «la vraie justice». Cette idée traverse les vingt-deux livres de son ouvrage De Civitate Dei; la seconde, que cette justice découle de la foi, qui est la cause du salut ${ }^{102}$. Il s'agit de bien comprendre cette différence majeure: Schmitt définit le souverain comme «celui qui décide de la situation exceptionnelle ${ }^{103}$. Il est donc question de cas de conflit et de décision relative à la détermination de l'intérêt public et de celui de l'État, la sûreté et l'ordre publics, le salut public ${ }^{104}$. Ces cas d'exception sont les cas d'extrême nécessité comme la menace pour l'existence de l'État. Seule cette situation, écrit Schmitt, rend actuelle la question de la souveraineté ${ }^{105}$. Selon le professeur O. Beaud, cette définition de la souveraineté, donnée par Schmitt, est révélatrice de son décisionnisme ${ }^{106}$. Et si son décisionnisme était d'une inspiration augustinienne, comme l'avait écrit le professeur $\mathrm{O}$. Beaud, il serait conséquent de tirer la conclusion que la pensée de saint Augustin conduirait à une apologie de la guerre dans la relation entre les États, puisqu'au nom de ce décisionnisme, Schmitt adopta une telle définition de la souveraineté qui implique une seule chose: décider de faire la guerre et détenir les moyens effectifs de la faire ${ }^{107}$. Or, saint Augustin n'a jamais parlé du droit naturel de l'État dans ce sens. Encore une fois, il s'agit pour saint Augustin de souligner le droit naturel de l'État d'exister face à l'Église. Contrairement à une idée bien répandue sur la doctrine de la guerre chez saint Augustin, selon laquelle le Père latin de l'Église adopta une conception de la guerre préventive qui aurait pu inspirer Schmitt, la lecture du chapitre XIII de son livre XIX nous conduit à une conclusion contraire. Voici ce que saint Augustin écrit au sujet de la paix universelle: «La paix de l'âme raisonnable, c'est en elle le parfait accord de la connaissance et de l'action. La paix entre l'homme mortel et Dieu est une obéissance réglée par la foi et soumise à la loi éternelle; celle des hommes entre eux, une concorde raisonnable. La paix d'une cité, c'est la même correspondance entre ses membres. La paix de la Cité céleste consiste dans une union très réglée et très parfaite pour jouir de Dieu, et du prochain en Dieu.»

On voit à travers ce passage la portée de la thèse de la doctrine de la guerre de saint Augustin. Celui-ci a une doctrine de la paix qui ne donne lieu à aucune théologie de la guerre d'aucune sorte. Dans son livre XIX en particulier, il développe sa doctrine de la paix et celle qui était perçue comme doctrine de la guerre. Il écrit aux chapitres XI et XII une apologie de la paix et non de la guerre préventive telle que Schmitt la conçoit. Il suffit de présenter trois citations de nos auteurs sur cette question. D'abord, saint Augustin estime que «même ceux qui font la guerre ne la font pas que pour vaincre, et pour parvenir glorieusement à la paix [...] Les guerres sont faites en vue de la paix [...] le véritable but de la guerre, c'est la paix, l'homme qui fait la guerre cherchant la paix, et nul ne faisant la paix pour avoir la guerre».

Le propos de saint Augustin s'inscrit dans une logique d'un devoir être moral. Ensuite, il attire notre attention sur une idée simple: "Le nom même de la Cité sainte, c'est-à-dire Jérusalem, est un nom mystérieux qui signifie vision de paix. Mais, comme on se sert aussi du nom de paix dans les choses de cette vie périssable, nous avons mieux aimé appeler vie éternelle la fin où la Cité de Dieu doit trouver son souverain bien.»

Enfin, il écrit sans ambiguïté « les bons s'ils sont vraiment bons ne combattent pas contre les bons: ceux qui se battent ce sont les méchants avec les méchants ou les méchants avec les bons » ${ }^{108}$. Et voici, en revanche, ce que Schmitt écrit au sujet de l'ennemi contre qui on rentre en guerre: «La justice de la guerre, lorsqu'elle est rapportée à la justa causa, comporte toujours à l'état latent une amorce de discrimination de l'adversaire injuste, et donc

99. L'auteur de cette thèse est le premier à considérer que son choix du vocable augustinisme politique n'est pas très pertinent. Il ne tient pas spécialement à le garder sinon faute d'un meilleur vocable, H.-X. Arquillière, L'augustinisme politique..., p. 19, note 2.

100. Ibid., p. 54

101. Ibid., p. 38-39.

102. Voir H. de Lubac, Théologies d'occasion, p. 264.

103. C. Schmitt, Théologie politique, p. 15.

104. Ibid., p. 16.

105. Ibid., p. 17 .

106. O. Beaud, préface, p. 112.

107. O. Beaud, préface. Nous avons déjà abordé la question de la guerre et de la paix chez saint Augustin à la fin du paragraphe $\mathrm{C}$ de la première partie de cette étude.

108. Saint Augustin, La Cité de Dieu (L. Jerphagnon (trad.), Paris, Gallimard (Pléiade), 200o), cité par A. Vanderpol dans La doctrine scolastique du droit de guerre, Paris, Pedone, 1925, p. 28. 
d'élimination de la guerre comme institution juridique. La guerre devient alors bien vite une simple action pénale, elle prend un caractère punitif, tous les graves dubia de la doctrine du bellum justum sont vite oubliés; l'ennemi devient simple criminel, et le reste, à savoir la privation des droits de l'adversaire et le pillage de ses biens, c'est-àdire la destruction du concept d'ennemi qui formellement présuppose toujours un justis hostis s'ensuit pratiquement tout seul.» ${ }^{109}$

Ainsi, il est assez simple pour Schmitt de considérer son ennemi comme un criminel dont l'anéantissement est une sorte de sanction pénale ${ }^{110}$ ! Si la détermination de l'ennemi est une question de décision et de volonté selon Schmitt, et si le «souverain est celui qui décide de la situation exceptionnelle», il est aisé d'en déduire que l'articulation entre décisionnisme, détermination de l'ennemi et doctrine de la guerre est définitivement établie. La conséquence de cette articulation est assez simple: par une décision fondée sur une appréciation subjective, on peut désigner l'ennemi dont l'anéantissement n'est que la stricte application de la loi. Le lexique guerrier de Schmitt n'a rien à voir avec celui du Père latin de l'Église, il témoigne plus d'un écart avec saint Augustin que d'une filiation: «l'éventualité effective d'un combat», "l'éventualité d'une lutte», "antagonisme suprême», "point extrême du conflit», «logique ultime de l'antagonisme», «degré extrême». Serait-il par pur hasard que Schmitt utilise les termes «État total», « ennemi total», ou encore «guerre totale»? Certaines interprétations des écrits de saint Augustin, notamment du livre XIX de La Cité de Dieu, considèrent qu'il y a là une légitimation de la guerre sainte et préventive. Pourtant, saint Augustin parla dans ce livre des persécutions injustes des chrétiens par les impies, celles que font les impies à l'église du Christ. Le contexte dans lequel il se situe est plutôt celui des luttes entre la chrétienté et l'agression du paganisme ou de l'hérésie. Il a mis l'accent dans ce livre surtout sur la paix et le besoin humain de la paix et non de la guerre ${ }^{111}$. En revanche, voici ce que notre penseur allemand a déclaré dans son ouvrage La Notion de politique: «La guerre, les hommes qui se battent, prêts à mourir, le fait de donner la mort à d'autres hommes qui sont, eux, dans le camp ennemi, rien de cela n'a de valeur normative, il s'agit au contraire de valeurs purement existentielles insérées dans la réalité d'une situation de lutte effective contre un ennemi réel et qui n'ont rien à voir avec quelques idéaux, programmes ou abstractions normatives. ${ }^{112}$
Mais il ajoute également qu' "aussi longtemps qu'un peuple existe dans la sphère du politique, il devra opérer lui-même la distinction entre amis et ennemis ${ }^{113}$.

Schmitt va plus loin: l'essence de l'existence politique d'un peuple dépend entièrement de sa capacité d'opérer une telle distinction ${ }^{114}$. Ne serait-il plus conséquent d'admettre que la confrontation avec l'ennemi total appelle la guerre totale et donc à une mobilisation totale au sein de l'État total schmittien? La guerre totale matérialise toutes possibilités théoriques que recelait l'articulation entre guerre et politique dans le décisionnisme politique de Schmitt. De même que l'État total fait tomber la frontière entre l'étatique et le social. Car si la société s'organise en État, si État et société doivent être principalement identiques, alors tous les problèmes sociaux et économiques deviennent immédiatement étatiques, et l'on ne peut plus distinguer entre des secteurs étatiques-politiques et des secteurs sociaux-apolitiques. Quant à la guerre totale, elle signifie, elle aussi, l'abolition de la distinction entre combattants et non-combattants, guerre militaire et non militaire. Elle conduit par sa conceptualisation même à l'engagement de toutes les ressources d'une nation dans la lutte contre l'ennemi afin de le détruire totalement. Ainsi, mais seulement ainsi, la guerre se réalise dans une entreprise d'extermination totale de l'ennemi dont l'identité est déterminée de manière subjective et hissée au rang de loi universelle qui appelle l'obéissance. La guerre naît de l'hostilité, celle-ci étant la négation existentielled'un autre être (l'Autre), elle va à l'encontre de l'enseignement chrétien qui repose sur la reconnaissance de l'Autre comme un être singulier, unique, et irremplaçable ${ }^{115}$. Or, quelques questions se posent: où est la paternité avec la doctrine de saint Augustin? Pourquoi ne pas poser plutôt les questions de savoir: qui nous a appris que le jugement humain subjectif pouvait hisser au rang de loi universelle? Où estce que nous avons lu des magnifiques pages de la philosophie du devoir et de l'impératif catégorique sinon chez Kant? Quel philosophe s'est opposé au droit de la résistance à l'oppression des lois injustes sinon Kant? Où est la proximité ou le lien entre ces idées et la vision augustinienne de la paix universelle et de la justice comme vertu de la république? Dans quel ouvrage saint Augustin a-til écrit qu'il faut «maltraiter l'ennemi et lui ôter tous ses droits pour parvenir à son anéantissement total»? Le chercheur qui étudie la généalogie de la pensée d'un auteur comme Schmitt ne devrait pas se contenter de rechercher une simple ressemblance sémantique, étymologique ou

109. C. Schmitt, Le Nomos de la Terre dans le droit des gens du Jus publicum europaeum [1950], L. Deroche-Gurcel (trad.), P. Haggenmacher (éd.), Paris, PUF, 2001, p. 123.

110. Voir en particulier ses deux ouvrages: La Notion de politique et Théologie politique.

111. Voilà ce que nous pouvons lire dans le chap. XII du liv. XIX: «Les guerres sont donc toujours faites en vue de la paix, même par ceux qui prennent plaisir à exercer leur vertu guerrière dans les combats; d'où il faut conclure que le véritable but de la guerre, c'est la paix".

112. Voir C. Schmitt, La Notion de politique..., p. 90.

113. Ibid., p. 91.

114. Ibid.

115. Voir les travaux du professeur J.-M. Trigeaud, et en particulier Humanisme de la liberté et philosophie de la justice, t. I et II, Bordeaux, Éditions Bière (Bibliothèque de philosophie comparée), 1990; Persona ou la Justice au double visage. 
conceptuelle de certaines notions transposées formellement dans un autre domaine. Si l'on se contentait de fonder la filiation intellectuelle et l'origine conceptuelle sur une analogie erronée, par exemple, entre la médiation chrétienne et la médiation étatique, ou entre l'hérétique et l'ennemi politique, et ainsi de suite, sans comprendre le rôle et la nature de ces concepts transposés, dans leur système d'origine, on commettrait une "erreur manifeste d'interprétation », par méconnaissance de la fonction originaire du concept que Schmitt a transposé hors de son contexte.

\section{Précautions de la recherche généalogique}

Au-delà de la problématique traitée, le développement précédent nous conduit à constater un phénomène qui caractérise la recherche en théorie constitutionnelle: le théoricien publiciste moderne n'essaie pas de résoudre la question de la dogmatique juridique en droit public par un recours, peut être à ses yeux, entaché de honte et ascientifique, à la métaphysique des métaphysiciens (considérée comme anti- ou ascientifique et irrationnelle), mais par un recours à la métaphysique des modernes ${ }^{116}$ dont les idées se rapprochent plus d'une certaine forme de positivisme philosophique déguisé (la métaphysique de Kant $\left.^{117}\right)$. Si Schmitt ne fait que transposer la structure formelle, ou le cadre vide, pour mettre à l'intérieur ce qui convient à son projet et à sa vision du droit et de la politique, Jacques Maritain, inspiré, a plutôt essayé d'éviter l'absolutisme de l'État, notamment après les trois expériences totalitaires européennes ${ }^{118}$.

Les sources chrétiennes ont pu jouer, pour certains auteurs, le rôle de «boîte à outils » dans la mesure où elles donnent des instruments de raisonnement. Il convient de souligner qu'à aucun moment, Schmitt n'avait accepté les réponses auxquelles il parviendrait s'il avait utilisé ces outils aux fins pour lesquelles ils ont été créés initialement. Or, il a fabriqué d'autres réponses, celles qui lui conviennent ou celles qui l'obsèdent, en laissant loin de vue l'essentiel: à savoir le projet, l'objectif, et la finalité pour lesquels ces outils et concepts ont été créés. D'où notre désaccord sur l'existence d'un quelconque augustinisme, politique ou juridique, chez Schmitt.

On l'aura peut-être compris: les inspirations attribuées à la doctrine catholique se limitent à la transposition formelle des concepts bruts sans oser adopter ni leur contenu, ni leur contexte. Cela aurait contrarié considérablement les idées politiques de Schmitt. Il s'agit de régler des problèmes théoriques politico-constitutionnels que la pensée politique n'a pu analyser qu'en faisant appel à d'autres disciplines métaphysiques, logiques ou philosophiques. Qu'il s'agisse de sa conception du droit et de sa théorie de la décision ou qu'il s'agisse de la théorie de la Constitution, les sources qui auraient pu inspirer certaines idées de Schmitt seraient une sorte de nationalisme autoritaire qui ne doit rien au christianisme, doublé d'un idéalisme allemand répandu à son époque. Serait-il si difficile aux chercheurs d'étudier la différence entre la métaphysique des positivistes tels que Kant et Descartes ${ }^{119}$ et la métaphysique des métaphysiciens tels que saint Augustin et Pascal $^{120}$ ? Affirmer une telle inspiration chrétienne de la pensée de Schmitt, c'est méconnaître la pensée chrétienne et les fondements mêmes du christianisme. Si les idées principales qui jalonnent ${ }^{121}$ les quatre essais (Théorie de la Constitution, Théologie politique, La Notion de politique, et La Dictature ${ }^{122}$ ) étaient examinées ici en confrontation aux sources chrétiennes, c'était pour déterminer la part de la pensée chrétienne dans ces idées. Tout examen de la généalogie intellectuelle d'un auteur devrait observer quelques précautions de recherche: si l'on constate avec la doctrine que des concepts philosophiques et théologiques ont été transposés de leur cadre et de leur contexte théologique à un autre contexte politique et constitutionnel, cela ne signifie pas que la source intellectuelle et doctrinale de Schmitt serait saint Augustin, dans la mesure où les idées et l'esprit de celui-ci ont été transformés et dénaturés pour en faire tout autre usage qui n'a strictement rien à voir avec leur source première. Le transfert est strictement formel comme nous l'avons déjà souligné (transfert du cadre vide et rien d'autre). D'ailleurs paradoxalement, on a reproché à Schmitt non pas le caractère augustiniste de sa doctrine, mais le caractère catholique thomiste de «la pensée d'ordre concert» inspirée de «l'esprit objectif» de Hegel comme l'avait bien noté David Cumin ${ }^{123}$.

116. Sur l'institution en tant que traduction matérielle chez Schmitt et le passage du transcendant invisible à l'empirique, de l'irrationnel au rationnel, voir S. Baume, Carl Schmitt, penseur de l'État..., p. 204-218.

117. Le système kantien annonçait dans l'introduction à la Critique de la faculté de juger, après la Critique de la Raison pure, l'impossibilité de la métaphysique traditionnelle et le développement d'un nouveau système métaphysique qui serait l'accomplissement de la philosophie rationnelle (même entreprise de Descartes). Ce nouveau système réconciliera la partie morale et la partie naturelle de la philosophie ou la morale et l'empirisme. Cette démarche s'inscrit dans la lignée des philosophies des Lumières.

118. Voir notre étude "Maritain et Kelsen, deux théories de la démocratie et de l'État pour l'Europe. "Confrontation" ", Droit prospectif: revue de la recherche juridique, vol. $33, \mathrm{n}^{\circ} 123,2008$, p. 1265-1298.

119. La distinction visée ici repose, d'une part, sur le rapprochement fait par J. Maritain entre les sources métaphysiques de Kant et celles de Descartes, et d'autre part, sur leur commune opposition à la métaphysique proprement dite, c'est-à-dire la métaphysique chrétienne aristotélicienne et thomiste. Voir une excellente analyse menée par J. Maritain sur cette question, Le Songe de Descartes, Paris, Éditions R.-A. Corrêa, 1932, p. 129-135.

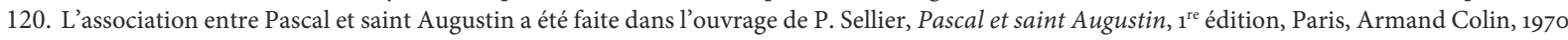
[réédition: Paris, Albin Michel, 1995].

121. Voir la présentation de l'œuvre de C. Schmitt dans la préface du professeur O. Beaud (préface, p. 14-113).

122. Il faut souligner que certains travaux de Schmitt cités dans cette étude ont été élaborés avant son adhésion au parti national-socialiste et donc avant son implication intellectuelle au nazisme.

123. D. Cumin, Carl Schmitt: biographie..., p. 149. 
Enfin, on ne saurait conclure sans mentionner l'importance pour le chercheur en matière de sécularisation de la pensée politique et constitutionnelle de connaître les combats politiques et intellectuels des auteurs dont il parle. Cela permet de nuancer certaines conclusions hâtives et erronées. Un seul exemple illustre notre propos pour chaque auteur. En ce qui concerne Schmitt, la polémique entre lui et le théologien Erik Peterson sur l'idée même d'une théologie politique dont les arguments sont développés dans leurs deux ouvrages (Théologie politique de Schmitt et Le monothéisme comme problème politique: une contribution à l'histoire de la théologie politique dans l'Empire romain de Peterson ${ }^{124}$ ). L'origine de l'opposition de Peterson aux thèses de Schmitt réside dans son refus d'utiliser le christianisme comme caution du nazisme. Lorsque les nazis prennent le pouvoir, ils cherchent à mettre au pas les Églises, Peterson s'élève contre l'instauration d'une Église du Reich en s'opposant à Schmitt et à sa conception de la "théologie politique». Il défend au contraire la thèse chrétienne selon laquelle le christianisme et la théologie ne doivent pas servir de caution à aucune entreprise politique de domination. Cette attitude courageuse l'entraîne également à redéfinir l'Église et à prendre parti, de manière très originale, sur la question de la nature et du rôle de la théologie. À cet égard, le constat fait par Sandrine Baume est exact: Schmitt fait sans cesse l'analogie entre l'Église et l'État, entre l'infaillibilité du pape et la souveraineté de l'État ${ }^{125}$, mais nous constatons aussi qu'il s'oppose ouvertement à saint Augustin lorsqu'il conteste la thèse de Peterson selon laquelle «la théologie de la paix de saint Augustin a liquidé toute théologie politique jusqu'à la fin des temps » ${ }^{126}$. Que faut-il d'autre pour marquer la rupture entre saint Augustin et Schmitt?

Au terme de cette série d'arguments, on peut conclure que si Schmitt récupère la cité de Dieu, ce n'est pas pour la faire vivre dans la cité terrestre, mais pour l'anéantir et l'exclure en l'incluant dans l'État. Sur le plan philosophique, et en particulier du point de vue de la philosophie personnaliste chrétienne, Schmitt l'évacua définitivement en méconnaissant l'homme en tant que personne et en confondant grossièrement l'individualisme contre lequel il lutta et le personnalisme chrétien étranger à sa doctrine. Le risque de la sécularisation schmittienne réside dans l'usage fait par le théoricien des concepts et outils intel- lectuels destinés à servir une autre cause et un autre type de rapports ${ }^{127}$. Que la philosophie qui anime la doctrine de Schmitt soit fondée sur des idées surnaturelles dont l'ancrage résiderait dans une certaine forme de métaphysique positiviste ou dans une variante du criticisme kantien $^{128}$, c'est bien possible. Mais prétendre qu'il s'agissait ici du christianisme, de théologie chrétienne ou pire encore d'augustinisme politique ou juridique au sens strict, c'est une erreur manifeste d'interprétation. Celle-ci résulte, d'une part, d'une confusion entre plusieurs acceptions de la métaphysique qui existaient avant et après les Lumières françaises et allemandes. La philosophie allemande postkantienne s'emploie à séculariser systématiquement la religion, contrairement à la philosophie française des Lumières qui pose le principe de la laïcité et qui coupe net entre l'État et la religion. Et d'autre part, cette erreur résulte aussi de la méconnaissance du christianisme et de la philosophie qui fonde la pensée de saint Augustin. Nous trouvons d'ailleurs la même méconnaissance, voire le lieu commun et l'a priori, autant chez Kelsen avec ses fausses idées sur le christianisme qu'on a pu lire dans son article sur la justice ${ }^{129}$, que chez Schmitt avec ses efforts de sécularisation.

Il était nécessaire sur le plan philosophique, mais aussi universitaire, de montrer les erreurs et les faiblesses de la thèse de l'augustinisme politique de Schmitt, pour ne pas la suivre sans vérifications ni précautions. On ne peut relever à la rigueur que le détournement de saint Augustin par Schmitt, mais en aucun cas une filiation, ou quelconque lien intellectuel d'aucune sorte. Schmitt a prétendu s'inspirer d'un grand nombre de théologiens et philosophes du passé, tout en les déformant et en les détournant. Le suivre dans son affirmation serait - me semble-t-il - une erreur scientifique soit par adhésion hâtive sans aller vérifier aux sources, soit par prédisposition intellectuelle à croire ce lieu commun contre les idées augustiniennes, ou encore par simple ignorance. En tout état de cause, les affirmations que nous avons récusées dans cette étude ne sauraient constituer une thèse doctrinale fondée et pertinente.

Enfin, cette étude devrait être complétée par d'autres qui porteraient principalement sur le rôle exact de l'idéalisme allemand sur des auteurs germanophones tels que Kelsen et Schmitt. Il s'agit de vérifier les différentes

124. E. Peterson, Le monothéisme comme problème politique: une contribution à l'histoire de la théologie politique dans l'Empire romain, Paris, Bayard, 2007.

125. S. Baume, Carl Schmitt, penseur de l'État..., p. 221-222.

126. C. Schmitt, Théologie politique, p. 152-153.

127. Des références importantes sur saint Augustin auraient dû attirer l'attention des juristes qui ont établi injustement des liens généalogiques entre Schmitt et saint Augustin par méconnaissance de l'œuvre du dernier. Nous en indiquons les références les plus importantes ici: L. Jerphagnon, Saint Augustin. Le pédagogue de Dieu, Paris, Gallimard (Découvertes, $\mathrm{n}^{\circ}$ 416), 2002; H.-I. Marrou, Saint Augustin et l'Augustinisme, Paris, Seuil (Maîtres spirituels, $\mathrm{n}^{\circ}$ 2), 1955; L'Église de l'Antiquité tardive, Paris, Seuil (Points Histoire), 1985; De la Connaissance historique, Paris, Seuil (Points Histoire), 1954; A. Mandouze, Saint Augustin. L'aventure de la raison et de la grâce, Paris, Études augustiniennes, 1968; E. Gilson, Introduction à l'étude de saint Augustin, $3^{\mathrm{e}}$ édition, Paris, Vrin, 1949; G. Bardy, Saint Augustin, l'homme et l'œuvre.

128. L'idéalisme allemand correspond à la fin des Lumières allemandes.

129. Sur les interprétations erronées de la conception chrétienne de la justice, on peut citer une illustration de cette méconnaissance dans certains écrits de H. Kelsen, What is justice? Justice, Law and Politics in the Mirror of Science, Berkeley - Los Angeles, University of California Press, 1957, p. 1-15. Voir aussi Essays in legal and moral Philosophy, Dordrecht, D. Reidel, 1973, p. 1-26, ainsi que son étude "Justice et droit naturel», Annales de philosophie politique, III, 1959, à titre d'exemples p. 15, 62, 72 et 86. 
formes d'influences de Kant et de l'idéalisme allemand en général sur la culture juridique et politique germanique et comment cette culture à son tour a influencé les juristes français. La question serait donc pourquoi la littérature française de la philosophie du droit public ne s'interroge que si rarement sur l'idéalisme comme une source des concepts juridiques, politiques ou constitutionnels employés par les juristes? À cette question, le sommeil dogmatique n'est pas une réponse. Cette étude n'a probablement pas suivi une démarche bien bernanosienne qui «s'efforce d'éveiller ceux qui dorment et à empêcher d'autres de s'endormir» sinon, peut-être, partiellement, en ce qu'elle serait « une besogne qui ne rapporte pas de grands profits, ni de grands honneurs, mais qui vous ferme beaucoup de carrières ${ }^{130}$. 